\title{
RETRACTED ARTICLE: Analysis of the role of breast dynamic nuclear magnetic resonance imaging in the treatment of breast tumors
}

\author{
Zhenghua Fei $^{1} \cdot$ Zhiqin Luo $^{1} \cdot$ Zhong Liu $^{1} \cdot$ Hongxia Xu ${ }^{2} \cdot$ Lijie Chen $^{2} \cdot{\text { Cunli } \mathrm{Ji}^{2}}^{2}$
}

Received: 21 February 2019 / Revised: 15 April 2019 / Accepted: 21 June 2019/

Published online: 7 August 2019

(C) Springer Science+Business Media, LLC, part of Springer Nature 2019

The Editor-in-Chief has retracted this article due to the authors' inability to provide documentation of approval from an ethics committee. Additionally, the article also shows evidence of authorship manipulation and peer review manipulation. The Editor-in-Chief therefore no longer has confidence in the validity of this article. The authors have not responded to any correspondence regarding this retraction.

Electronic supplementary material The online version of this article (https://doi.org/10.1007/s11042-01907913-6) contains supplementary material, which is available to authorized users.

\section{Zhenghua Fei}

feizhenghua@126.com

1 Department of Radiology, Huzhou Maternity \& Child Healthcare Hospital, Huzhou 313000, China

2 Breast Department, Huzhou Maternity \& Child Healthcare Hospital, Huzhou 313000, China 